\title{
Ontology-Driven, Adaptive, Medical Questionnaires for Patients with Mild Learning Disabilities
}

\author{
Ryan Colin Gibson ${ }^{1[0000-0002-0425-1486]}$, Matt-Mouley Bouamrane ${ }^{2[0000-0002-1416-751 X]}$ and \\ Mark D. Dunlop ${ }^{1}$ [0000-0002-4593-1103] \\ ${ }^{1}$ University of Strathclyde, Glasgow, United Kingdom \\ ${ }^{2}$ University of Edinburgh, Edinburgh, United Kingdom \\ ryan.gibsonestrath.ac.uk
}

\begin{abstract}
Patients with Learning Disabilities (LD) have substantial and unmet healthcare needs, and previous studies have highlighted that they face both health inequalities and worse outcomes than the general population. Primary care practitioners are often the first port-of-call for medical consultations, and one issue faced by LD patients in this context is the very limited time available during consultations - typically less than ten minutes. In order to alleviate this issue, we propose a digital communication aid in the form of an ontology-based medical questionnaire that can adapt to a patient's medical context as well as their accessibility needs (physical and cognitive). The application is intended to be used in advance of a consultation so that a primary care practitioner may have prior access to their LD patients' self-reported symptoms. This work builds upon and extends previous research carried out in the development of adaptive medical questionnaires to include interactive and interface functionalities designed specifically to cater for patients with potentially complex accessibility needs. A patient's current health status and accessibility profile (relating to their impairments) is used to dynamically adjust the structure and content of the medical questionnaire. As such, the system is able to significantly limit and focus questions to immediately relevant concerns while discarding irrelevant questions. We propose that our ontology-based design not only improves the relevance and accessibility of medical questionnaires for patients with LDs, but also provides important benefits in terms of medical knowledge-base modularity, as well as for software extension and maintenance.
\end{abstract}

Keywords: OWL ontologies, Adaptive questionnaire, Intelligent Software Development, Knowledge-Base Modularity, Human-Computer Interaction, Accessibility, Digital Communication Aids, Learning Disabilities

\section{$1 \quad$ Introduction}

Computer-based medical Information Collection Systems (ICS) can provide a range of benefits in comparison to conventional data extraction practices such as face-to-face interviews. These benefits include more accurate, structured and detailed data [1]; as well as allowing medical professionals to focus on providing patient-centered care, rather than conducting data collection tasks. ICS can also play an important role with regards to 'hard to reach' populations, such as individuals with learning disabilities 
(LDs). For example, medical professionals remain undertrained on the health and communication needs of patients with LDs [2], and this affects their ability to diagnose conditions that are commonly overshadowed within the LD population. In contrast, these symptoms may be embedded in ICS (such as [3-5]) that dynamically adjust to a patient's individual medical context, therefore identifying such conditions on a more consistent basis. Furthermore, systems such as [6,7] can present information to people with LDs in the format most suited to their own needs, thus increasing their ability to communicate about their health.

As such, adaptive ICS are particularly useful for patients with LDs since the medical conditions and specific needs of this population are heterogeneous and wide-ranging meaning a 'one-size-fits-all' model is simply unfeasible. Consequently, developing a multitude of questionnaires to cater for every possible scenario would be unmanageable and would not scale $[6,7]$.

In this paper we expand upon previous research on adaptive medical questionnaires (primarily $[3-5,8]$ ), by combining the use of 2 distinct ontologies into a single system, so that the proposed ICS can adapt to both the medical and accessibility needs of a patient with mild LD. An ontology is a formal description of a domain that models a set of concepts within this domain, as well as the relationships that exist between them. They have an important role in the healthcare as they enable complex concepts, such as patient data, to be captured and reused in a generic way.

Our system first mitigates the potential accessibility barriers experienced by a patient by mapping their cognitive and physical impairments to alterations in the user interface (e.g. changing the background color or prioritizing the use of audio feedback). A second medical ontology is then used to dynamically change the structure and content of a medical questionnaire so that it limits and focuses its scope to questions that are deemed immediately relevant to the patient's health status.

The remainder of this paper is structured as follows: we present the background and related work in the next section 2; the methodology in section 3; the ontology development in section 4 , the adaptive engine in section 5 and conclude with a scenario-based evaluation in section 6 .

\section{Background \& Related Work}

\section{Learning Disabilities}

In this work, we consider "learning disability" using the World Health Organization (WHO) definition": "they have a significantly reduced ability to understand new or complex information and to learn and apply new skills. This results in a reduced ability to cope independently and begins before adulthood with a lasting effect on development." LDs can also have a significant impact on an individual's capacity to interact with digital technologies as is discussed in depth in $[6,7]$.

\section{Adaptive Questionnaires}

\footnotetext{
${ }^{1}$ WHO: Definition: intellectual disability, https://bit.ly/2qFOwFX (2010)
} 
Bouamrane et al. were the first to propose the use of ontologies to drive the adaptive behavior of medical questionnaires during preoperative assessment [3-5]. Their ontology-based questionnaire updates its structure depending on the input received from the patient, thus removing questions that are of no relevance to the risk assessment while expanding on those deemed to be important. The system can thus capture finer-grained information providing it is relevant to the patient's specific medical context.

Benmimoune et al. [8] subsequently extended this framework to implement questionnaires which are not 'hard-coded' to a specific domain. They proposed a further ontology that gives meaning to a specific question by relating it to a concept within a particular healthcare domain such as "digestive surgery". The patient's interactions with the system (i.e. the questions presented / answers received) are also captured via an Interrogations History Ontology. This process is facilitated by a questionnaire engine (similar to that in [3-5]) which interprets the structure of the Questionnaire Ontology and stores the results in the Interrogations History Ontology.

\section{Accessible Interfaces}

With regards to the use of ontologies and knowledge-base to address the accessibility requirement of users with additional needs, Yesilada et al. [9] developed a semi-automated annotation tool that uses an ontology to translate web elements into "travel" concepts. These concepts assist users with visual impairments when navigating internet content. Obrenovic et al. [10] also employed ontologies to assist researchers in the creation of multimodal interfaces. Three sets of logically related ontologies were developed to capture basic concepts that may affect how an interface is utilized, including: the computing environment; the capabilities of the user; and the context of the user's surroundings. A fourth ontology is then used to import and connect the concepts from these domains to support the definition of interaction on multiple levels. Developers may then view how their design choices impact various human factors. Castillejo et al. also proposed an ontology that captures 3 sets of characteristics: the user's characteristics, the environment's characteristics, and the device's characteristics [11]. Rule sets are then executed to enact adaptions to a mobile device based on these characteristics.

Karim and Tjoa $[12,13]$ proposed using ontologies to formally describe a mapping between a user's impairments and the available interface characteristics (e.g. low visual acuity to text size). This was achieved via a class-subclass hierarchy and the use of description logic statements to compute automatic interface adaptions. Finally, in contrast to the previous approaches, Marino et al. [14] proposed a model which focuses on enhancing a user's capabilities as opposed to mitigating the impact of disabilities.

\section{$3 \quad$ Methodology}

Our system consists of 3 entities, as shown in Fig. 1: an ontology to model the accessibility needs of the patient; a second ontology to model the medical needs of the patient; and a Java Adaptive Engine to accept user input and interact with the ontologies as appropriate. We made a deliberate decision to separate the 2 ontologies to ensure they may be used as stand-alone resources and can be updated and maintained separately, 
which is considered as best-practice design in ontology and software engineering [15]. Both the accessibility preferences and medical questionnaire ontologies were modelled using the Web Ontology Language (OWL), and the Protégé-OWL development tool [16]. The java adaptive engine was implemented using the OWL API [17].

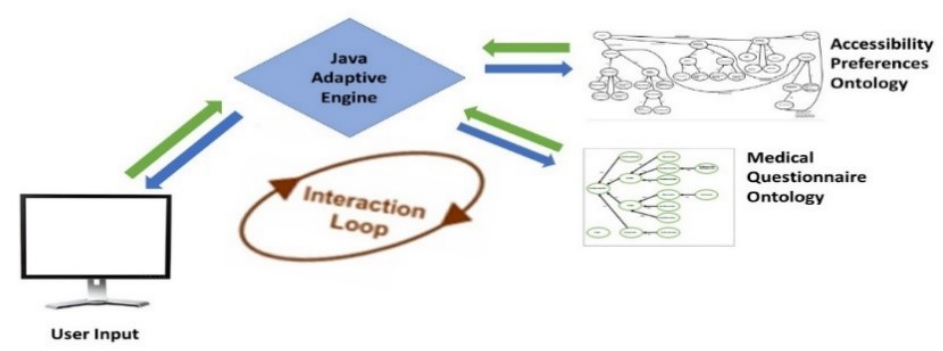

Fig. 1. System architecture for the adaptive questionnaire.

\section{Accessibility \& Medical Ontology}

The primary intention of the accessibility ontology is to model common impairments experienced by people with LDs (both physical and cognitive) and map these to potential adaptations in the interface employed. We identified and modelled these impairments using the International Classification of Functioning, Disability and Health (WHO-ICF) framework proposed by the World Health Organization ${ }^{2}$. Forward engineering was then used to semantically link these impairments to appropriate changes in the interface based on the Web Content Accessibility Guidelines ${ }^{3}$. This ontology requires the user to input their accessibility needs via a questionnaire (as opposed to an automated process that utilizes other resources) meaning it should be completed in conjunction with a carer or care assistant (e.g. practice nurse).

We used published guidelines regarding the health needs of people with LDs in order to design the questions to be embedded within the ICS; specifically, the "Learning Disability Health Toolkit"4 (LDHT) as it contains information on the most common symptoms experienced by LD patients. We used forward engineering to model the medical symptoms contained within the LDHT, as well as their relationship to other body parts or conditions in the medical ontology. We began by modelling the symptoms related to a single condition contained within the toolkit and iteratively refined the emerging properties as further conditions were added. The concepts were modelled as classes and subclasses, instead of instances, to aid in maintenance as we expect such concepts to change frequently as new guidelines are released. Since the needs of people with LDs differ dramatically from other populations, we were unable to identify (and therefore reuse) appropriate conceptualizations that met our goals. As such, it was necessary to develop the 2 ontologies described in the next section.

\footnotetext{
${ }^{2}$ https://www.who.int/classifications/icf/en/

${ }^{3} \mathrm{https} / / / \mathrm{www} . \mathrm{w} 3$. org/WAI/standards-guidelines/wcag/

${ }^{4}$ Turning Point: Learning Disability Health Toolkit, https://bit.ly/2JgecS0 (2016)
} 


\section{Java Adaptive Engine}

Rather than utilizing a reasoner to classify the behavior of the ontologies, we have developed a rule-based Java Adaptive Engine to decouple the handling of the user-input and the traversal of the ontologies from the actual ontologies. This also promotes convenient maintainability as changes can be made to the questionnaires without affecting the Java Adaptive Engine and vice-versa, allowing for lesser system complexity and higher modularity as recommended in [15].

\section{Ontologies Development}

\subsection{Medical Questionnaire Ontology}

The ontology aims to model two distinct aspects: (1) the structure of the questionnaire and (2) the adaptive behavior of the questionnaire. These fundamental principles are based on previous work [3-5], and have been adapted to cater to the concepts that emerged whilst modelling the conditions contained in the LDHT. A high-level overview of the developed classes may be found in Fig.2.

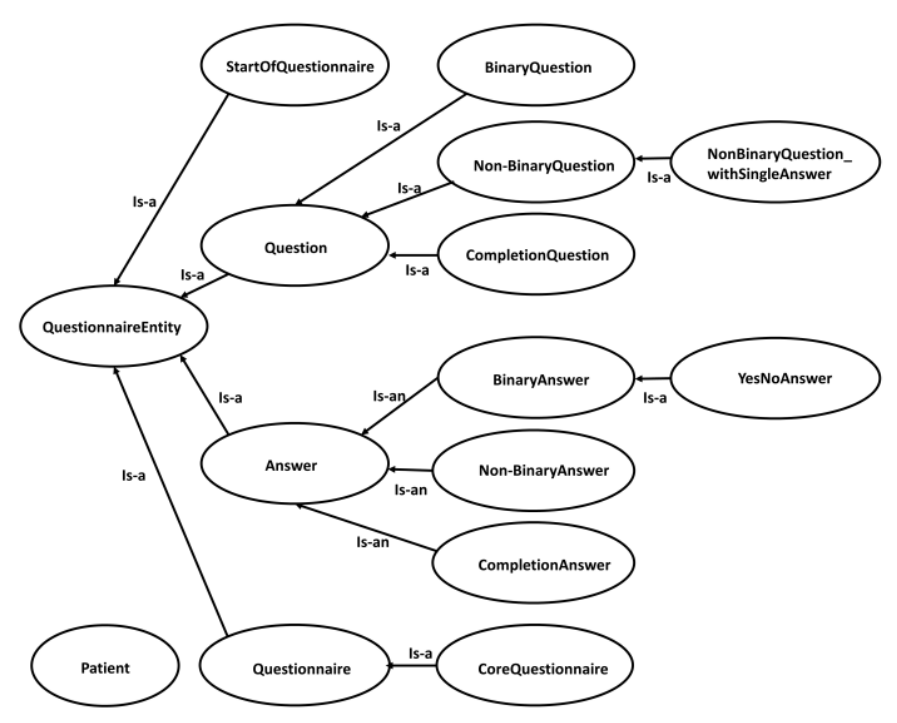

Fig. 2. Overview of the classes contained in the medical questionnaire ontology.

- Questionnaire: Comprised of thematically related Question classes.

- CoreQuestionnaire: Comprised of Question classes that are used to determine whether the primary Questionnaire's (equating to the body parts and conditions found in the LDHT) are presented to the patient. All Question's contained within are adaptive thus heavily restricting the number of Questionnaires parsed.

- StartOfQuestionnaire: Points to the Questionnaire class containing the first Question to be presented to the user - primarily CoreQuestionnaire, see section 5. 
- Question: Captures the information used to determine the runtime behavior of the questionnaire implementation. This includes: the set of possible Answers for a Question; and the set of potential actions that may occur upon receiving user input. 3 types of Questions are included: (1) BinaryQuestion provides exactly two Answers to the user, with the option to select one of these Answers. (2) NonBinaryQuestion presents 3 or more Answers, with NonBinaryQuestion_withSingleAnswer permitting the user to select just one of these Answers. (3) Finally, CompletionQuestion requires the user to input free text when answering Questions that have no defined Answers. All Questions are characterized by a questionContent property to display the question text and a questionPriority property to determine the order in which the Questions contained within a Questionnaire are presented.

- Answer: Mirrors the Question classes whilst encapsulating the information required by the user interface to display the Answer i.e. an answerContent property.

- Patient: Encapsulates the patient's personal information (gender, age, impairments) which facilitates the restriction of a specific Question or Questionnaire.

\section{Medical Questionnaire Properties}

Object properties are fundamental in defining both the structure of the questionnaire implementation and its run-time behavior. As such, two main sets of properties have been defined, structural and adaptive, and these are described in Table 1 using the acronyms $\mathrm{S}$ and A respectively. Examples of their use are provided in section 5.

Table 1. Object Properties included in Medical Questionnaire Ontology.

\begin{tabular}{|c|c|c|c|c|}
\hline Property & Type & Domain & Range & Description \\
\hline $\begin{array}{l}\text { containsQuestionA- } \\
\text { bout }\end{array}$ & $\mathrm{S}$ & $\begin{array}{l}\text { Question- } \\
\text { naire }\end{array}$ & Question & $\begin{array}{l}\text { Determines which Questions are } \\
\text { contained within a Questionnaire }\end{array}$ \\
\hline hasExpectedAnswers & $\mathrm{S}$ & Question & Answer & $\begin{array}{l}\text { Links a Question class to its Answer } \\
\text { classes }\end{array}$ \\
\hline $\begin{array}{l}\text { hasAlwaysRelat- } \\
\text { edQuestion }\end{array}$ & $\mathrm{S}$ & Question & Question & $\begin{array}{l}\text { Links two Question classes provided } \\
\text { one is always followed by the other. }\end{array}$ \\
\hline ifAnswerToThisQues & A & Question & Answer & Declares Question is adaptive. Links \\
\hline tionIs & & & & $\begin{array}{l}\text { Question to further Question classes } \\
\text { depending on the Answer received. }\end{array}$ \\
\hline thenGoToQuestion & A & & Question & $\begin{array}{l}\text { Links a follow-up question to a spe- } \\
\text { cific answer. }\end{array}$ \\
\hline $\begin{array}{l}\text { hasAssociatedQues- } \\
\text { tionnaire }\end{array}$ & A & Question & $\begin{array}{l}\text { Ques- } \\
\text { tionnaire }\end{array}$ & $\begin{array}{l}\text { Links a Question to a follow-up } \\
\text { Questionnaire. }\end{array}$ \\
\hline
\end{tabular}

Three specialized adaptive properties have also been defined to restrict the presentation of Questions based on the user's age (onlyIfAgeIs), sex (onlyIfSexIs), and impairments (onlyIfImpairmentIsNotApplicable). The latter depends on the information extracted from the Accessibility Preferences Ontology described in section 4.2. 


\subsection{Accessibility Ontology}

A wide range, and combination of, impairments must be addressed to ensure the system is accessible to the LD population. The accessibility preferences ontology achieves this by extracting the cognitive/physical impairments experienced by the patient, before mapping these to a model of interface changes that mitigate their effect (similar to [12]). A high-level overview of the ontologies structure may be found in Fig. 3. Its basic composition is similar to that of the medical questionnaire ontology described in section 4.1, with adjustments being made to capture the concepts included in the WHO-ICF framework. The new classes that emerged as a result of this process are as follows:

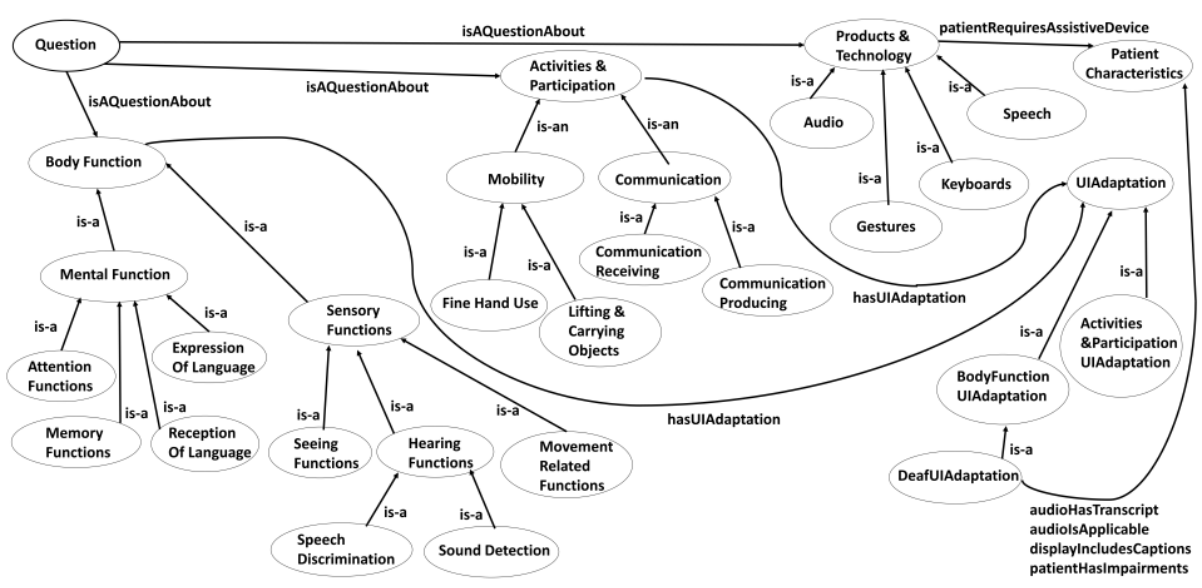

Fig. 3 Overview of the Accessibility Preferences Ontology. The hearing functions concept has been extended to demonstrate the effect a UIAdaptation may have on PatientCharactersistics.

- BodyFunctions: Captures the potential options (impairments) that may be presented to the user related to an individual's functioning of the body. This includes both mental functions, and sensory functions. An impairmentDescription annotation is used to describe the impairment in a textual format.

- ActivitesAndParticipation: Essentially the same as BodyFunctions except that it captures impairments that may affect an individual's ability to complete everyday tasks. This includes mobility and communication.

- ProductsAndTechnology: Captures the potential assistive devices required by the user to operate digital technologies. Such devices were extracted from [14] and grouped under the following concepts: Audio, Gestures, Keyboard and Screen.

- UIAdaptation: Models the interface adaptations that should occur once the user has indicated that they have an impairment or that they require an assistive device.

- PatientCharacteristics: Encapsulates the individual's user interface preferences, which have been previously captured via the UIAdaptation classes.

\section{Accessibility Ontology Properties}


Two sets of properties have been defined: object properties that determine the structure and run-time behavior of the questionnaire; and data properties that capture the individual's user interface preferences. Table 2 contains a description of the newly developed object properties, with examples of their use being found in section 5 .

Table 2. Object Properties included in Accessibility Preferences Ontology.

\begin{tabular}{|c|c|c|c|}
\hline Property & Domain & Range & Description \\
\hline $\begin{array}{l}\text { isAQuestionA- } \\
\text { bout }\end{array}$ & Question & & $\begin{array}{l}\text { Links a Question to a relevant option class i.e. a } \\
\text { subclass of BodyFunctions, ActivitiesAndPar- } \\
\text { ticipation, or ProductsAndTechnology }\end{array}$ \\
\hline $\begin{array}{l}\text { hasUIAdapta- } \\
\text { tion }\end{array}$ & & $\begin{array}{l}\text { UIAda- } \\
\text { patation }\end{array}$ & $\begin{array}{l}\text { Links an impairment i.e. a subclass of BodyFunc- } \\
\text { tions or ActivitiesAndParticipation to an appro- } \\
\text { priate UIAdaptation. }\end{array}$ \\
\hline
\end{tabular}

In addition, the ifAnswerToThisQuestionIs and thenGoToQuestion properties found in section 4.1 are also included and operate in the same manner. To capture the individual's user interface preferences, we have extended the list of user characteristic data properties in [11] (see table 3). This enables us to cater to additional conditions commonly experienced by people with LDs.

Table 3. PatientCharacteristics data properties extended from [11].

\begin{tabular}{|c|c|c|}
\hline Subclass & Property Name & Description \\
\hline Audio & audioHasTranscript & $\begin{array}{l}\text { A Boolean value that describes whether an accompa- } \\
\text { nying transcript should be provided in addition to au- } \\
\text { dio feedback. }\end{array}$ \\
\hline \multirow[t]{4}{*}{ Interface } & $\begin{array}{l}\text { interfaceEnablesScroll- } \\
\text { ing }\end{array}$ & $\begin{array}{l}\text { A Boolean value that indicates whether scrolling is } \\
\text { enabled. }\end{array}$ \\
\hline & interfaceEnablesSwiping & $\begin{array}{l}\text { A Boolean value that captures whether swiping is en- } \\
\text { abled. }\end{array}$ \\
\hline & interfaceTouchStrategy & $\begin{array}{l}\text { This property models the preferred touch input } \\
\text { method with the following possibilities: "default" and } \\
\text { "end-tap". }\end{array}$ \\
\hline & interfaceTracksAttention & $\begin{array}{l}\text { A Boolean value which indicates whether an eye- } \\
\text { tracker may be utilized to determine if the system is in } \\
\text { possession of the user's attention. }\end{array}$ \\
\hline \multirow[t]{2}{*}{ Patient } & patientHasImpairments & A list of impairments that affect the individual. \\
\hline & $\begin{array}{l}\text { patientRequiresAssis- } \\
\text { tiveDevice }\end{array}$ & $\begin{array}{l}\text { A list of assistive devices required by the individual to } \\
\text { operate digital technologies effectively. }\end{array}$ \\
\hline \multirow[t]{3}{*}{ View } & viewIncludesCaptions & $\begin{array}{l}\text { A Boolean value indicating whether videos should in- } \\
\text { clude captions. }\end{array}$ \\
\hline & viewIncludesGIFS & $\begin{array}{l}\text { A Boolean value describing whether GIFs are appro- } \\
\text { priate to the individual. }\end{array}$ \\
\hline & viewIncludesProgess & $\begin{array}{l}\text { A Boolean value which captures whether the individ- } \\
\text { ual's progress should be recorded and returned. }\end{array}$ \\
\hline
\end{tabular}




\section{System Implementation - Java Adaptive Engine}

\section{$5.1 \quad$ Java Engine}

As shown in Fig. 1, the Adaptive Engine is decoupled from the underlying questionnaire models. Consequently, we had a significant amount of discretion regarding the implementation of the engine, and ultimately chose to develop the questionnaire as a stack, similar to the approach adopted by Bouamrane et al. [3-5].

The engine first calls the method required to traverse the Accessibility Preferences Questionnaire Ontology and carries out the following 5 stage process: (1) the initial Question classes are loaded into the stack in order of priority. (2) The Question at the top of the stack is popped and presented to the patient, along with the potential options that the user may select from. These options are identified via the direct subclasses of the object contained in the current Question's "isAQuestionAbout" superclass. As such, they may be a subclass of ActivitesAndParticipation, BodyFunction, or ProductsAndTechnology. (3) An appropriate Answer is extracted from the user and subsequently mapped to changes in the interface via the filler contained in the selected $A n$ swer's "hasUIAdaption some UIAdaptation" superclass. The annotation properties held in the UIAdaptation class are then used to update those held in PatientCharacteristics.

(4) The engine then checks to see if the current Question is adaptive i.e. whether it is a subclass of "(ifAnswerToThisQuestionIs some Answer) and (thenGoToQuestion some Question). If the Question is not adaptive, or the input received from the user does not trigger further questions, the system moves on to stage 5. Otherwise, an additional Question is added to the top of the stack via the "thenGoToQuestion some Question" superclass. (5) Steps 2 to 5 are repeated until the stack becomes empty.

The Java Engine then calls the method used to traverse the Medical Questionnaire Ontology and subsequently passes in the information held in PatientCharacteristics' patientHasImpairments data property. This parameter is used to update the hasImpairments property contained in the Medical Questionnaire's Patient class, which facilitates the restriction of Questions based on the user's physical or cognitive disabilities. The following 5 steps are then carried out.

(1) the starting Questionnaire is identified by examining StartOfQuestionnaire and extracting the filler from its superclass "hasAssociatedQuestionnaire some Questionnaire". (2) The Questionnaire's "containsQuestionAbout some Question" superclass is then examined with all direct subclasses of the filler being added to the stack in order of priority, provided they satisfy all restrictions placed on it e.g. a Question may not be added if it is a subclass of "onlyIfSexIs some Female" and the patient is male.

(3) The Question at the top of the stack is popped and presented to the patient along with the set of possible answers the user may select from. These options constitute the direct subclasses of the filler included in the Question's "hasExpectedAnswer some Answer" superclass. (4) Once an appropriate Answer has been received from the patient, the Java engine stores the Question/Answer pairing and subsequently checks to see if the current Question is adaptive i.e. whether its superclasses includes 
"ifAnswerToThisQuestionIs some Answer." If the Question is not adaptive or the Answer received by the user does not trigger its adaptive properties, then the system moves on to stage 5. If the current Question is adaptive and requires a single Question to be added to the stack, then this is pushed to the top via "thenGoToQuestion some Question", provided it meets all restrictions placed on it. If multiple Questions are required to be added e.g. those contained in a Questionnaire, then this is done in a similar process to that described in stage 2. (5) Stages 3-5 are repeated until the stack becomes empty.

\subsection{Dynamic Changes to Stack}

We will now present an example of how the Medical Questionnaire stack reacts to the patient's input, to show the importance of CoreQuestionnaire and onlyIfImpairmentIsNotApplicable in reducing the number of irrelevant Questions presented.

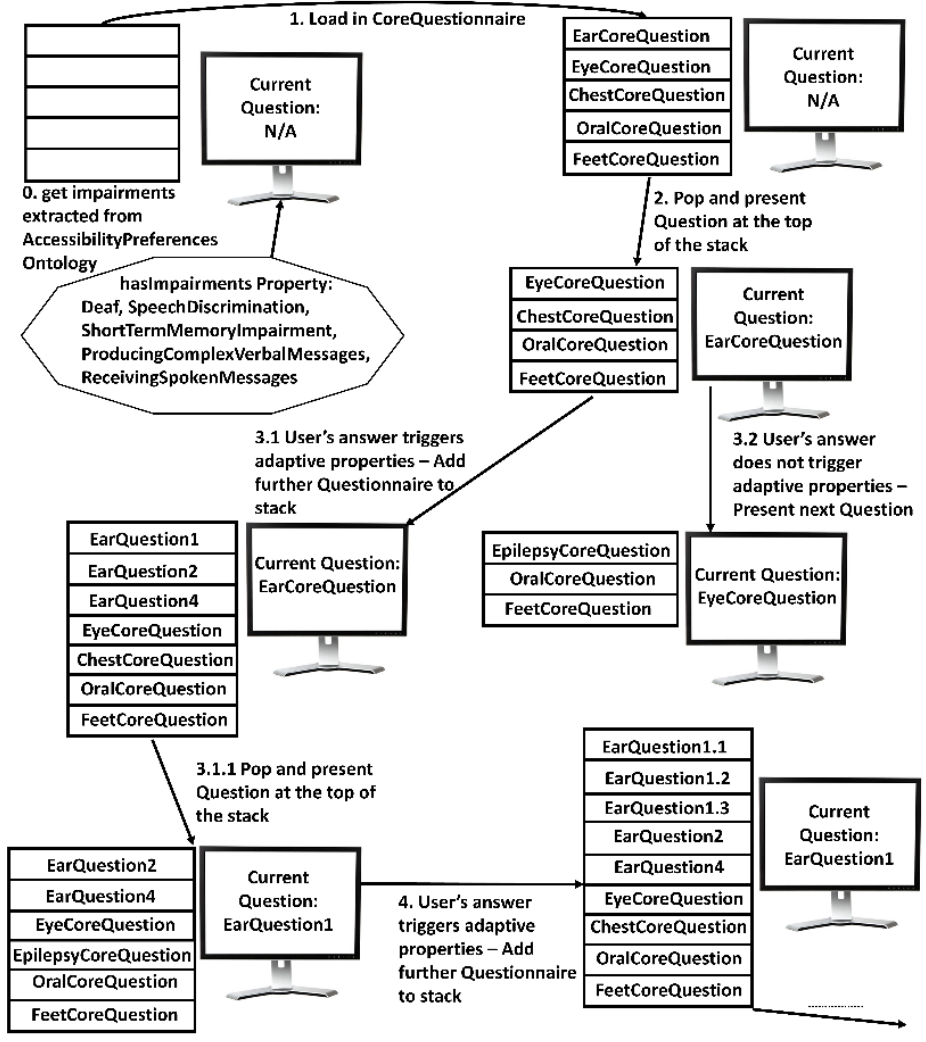

Fig. 4. Changes to questionnaire stack based on user's input \& accessibility needs.

The initialization phase is shown in step 0 of Fig. 4 and involves the hasImpairments property of the Patient class being updated with the impairments identified by the Accessibility Preferences ontology. The Java Engine then pushes all Questions contained in CoreQuestionnaire (step 1) to the stack in order or priority, since this is identified as the starting Questionnaire. CoreQuestionnaire encapsulates the Question classes that 
link to further Questionnaires containing queries on specific body parts or conditions. All Questions are adaptive meaning an entire Questionnaire can be bypassed based on a single response received from the patient. For example, in step 3 of Fig. 4 the user is required to answer the current Question displayed - in this case EarCoreQuestion. The questionContent annotation attached to this class is presented on the screen along with the possible answers. If the user selects the option "No", the system simply presents the next Question at the top of the stack, see step 3.2 in Fig. 4. Consequently, the class EarQuestionnaire is never parsed by the Java Engine or presented to the user.

Step 3.1 in Fig. 4 demonstrates what occurs if the patient's answer triggered the adaptive properties of EarCoreQuestion. All Questions contained in EarQuestionnaire is added to the stack except from EarQuestion3. EarQuestion3 is not parsed as it is a subclass of "onlyIfImpairmentIsNotApplicable some Deaf" and the condition "Deaf" is included in Patient's hasImpairments property. Questions that are not a subclass of CoreQuestionnaire may also cause additional Questions/Questionnaires to be added to the stack, as shown in step 4 of Fig. 4.

\section{Scenario Based Evaluation}

In a similar approach to [11], we have used scenarios to demonstrate the scope of adaptation that occurs when the Medical Questionnaire Ontology responds to the accessibility and medical needs of users. A more empirical evaluation was not appropriate at this stage due to the lack of available clinical alternative and augmentative communication applications for people with LDs. Previous research such as [6,7] has focused on extracting design requirements from experts, as opposed to target stakeholders; thus, embedding the questionnaire within such technologies could negatively influence the results obtained, if the user interface is inappropriate for the participants needs. Currently, the Medical Questionnaire Ontology is populated with 110 Questions across 9 distinct Questionnaires capturing conditions of the mouth, feet, chest, ears and eyes, as well as the patient's mental wellbeing, toiletry habits, weight trends, and general health.

\section{Scenario 1:}

Jane currently works for a national advocacy charity. The left-hand side of her vision is impaired meaning she finds it difficult to interact with applications that have been developed in the standard, justified format. Jane has a slight motor impairment; however, this does not affect her ability to interact with digital technologies on an everyday basis. Nonetheless, when she becomes tired her touch accuracy reduces significantly, at which point she prefers to interact with the user interface via speech.

Table 4 includes the most relevant sections of Jane's user interface model proposed by the Accessibility Preferences Ontology. The main adaptation to the default interface is captured via the viewHasPageLayout property, which aligns the elements to the righthand side of the screen. Since her visual acuity is unaffected the default text-size is reduced to assist this process. Regarding Jane's motor impairments, the model has suggested that touch input should only register once an action has been completed, whilst audio input is also a recommended as an interaction modality. 
Jane's Medical Needs: Jane has recently secured a promotion at the advocacy charity meaning her responsibilities have increased substantially over the last few weeks. This increased workload is becoming overwhelming and has had a significant impact on 3 areas of Jane's life - her social routine, relationship with peers, and mental wellbeing. She is currently experiencing the following primary symptoms: difficulty sleeping due to heightened stress and anxiety; a decrease in attentiveness; irritation; and isolation.

In this instance, just one of the Questions contained in CoreQuestionnaire has its adaptive properties triggered - MentalWellbeingCoreQuestion. Consequently, only the mental wellbeing Questionnaire is presented to Jane in addition to the initial 9 Questions contained in the CoreQuestionnaire. The mental wellbeing Questionnaire includes a total of 15 Questions of which 6 are dependent on the adaptive properties of 2 separate Questions - SocialRoutineQuestion and SleepingRoutineQuestion. These adaptive properties are triggered, meaning a total of 23 Questions from a possible 110 $(20.91 \%)$ are presented to Jane.

Table 4. Important sections of the proposed user interface model for scenarios 1 and 2.

\begin{tabular}{|c|c|c|c|}
\hline \multicolumn{2}{|c|}{ Scenario 1} & \multicolumn{2}{|c|}{ Scenario 2} \\
\hline Property & Value & Property & Value \\
\hline viewHasPageLayout & Right-aligned & viewHasTextSize & 14 \\
\hline $\begin{array}{l}\text { patientRequiresAssis- } \\
\text { tiveDevice }\end{array}$ & SpeechRecognition & viewIncludesCaptions & True \\
\hline $\begin{array}{l}\text { interfaceAcceptsAu- } \\
\text { dioInput }\end{array}$ & True & $\begin{array}{l}\text { patientRequiresAssis- } \\
\text { tiveDevice }\end{array}$ & ScreenMagnifier \\
\hline viewHasTextSize & 10 & $\begin{array}{l}\text { interfaceAcceptsAu- } \\
\text { dioInput }\end{array}$ & True \\
\hline $\begin{array}{l}\text { interfaceTouchStrat- } \\
\text { egy }\end{array}$ & End-tap & $\begin{array}{l}\text { interfaceTrack- } \\
\text { sUserAttention }\end{array}$ & True \\
\hline $\begin{array}{l}\text { patientHasImpair- } \\
\text { ments }\end{array}$ & $\begin{array}{l}\text { ComplexMotorFunc- } \\
\text { tions, LeftFieldLoss, } \\
\text { TappingAccuracy }\end{array}$ & $\begin{array}{l}\text { patientHasImpair- } \\
\text { ments }\end{array}$ & $\begin{array}{l}\text { Deaf, SpeechDiscrim- } \\
\text { ination, ShortSighted, } \\
\text { AttentionDeficit, } \\
\text { ShortTermMemory, } \\
\text { Producing\&Receiv- } \\
\text { ingVerbalMessages }\end{array}$ \\
\hline & & audioIsApplicable & False \\
\hline & & viewIncludesProgress & True \\
\hline
\end{tabular}

\section{Scenario 2:}

John is deaf and therefore has a dependence on visual methods to receive information. Despite this reliance, he is short-sighted and finds it difficult to read small text. In addition, the patient's LD affects their capacity to understand obscure or abstract information and significantly impedes their attention span and short-term memory. He is able to express simple concepts - such as yes or no - via the use of speech yet struggles to convey more complex words/sentences coherently. 
The primary adaptations that occur (see Table 4) relate to the user's inability to detect sounds. The audioIsApplicable property states that sound is not a viable method used to provide feedback. Despite this, John has indicated that he is able to use speech to communicate simple needs, hence why the interfaceAcceptsAudioInput value is True. viewIncludesCaptions expresses the need to provide captions alongside any media content. Several adaptations also occur to combat John's short attention span, along with an increase in text size to overcome his short sightedness.

John's Medical Needs: Regarding John's current health status, he has been confined to his bed over the last few days with a high fever and a feeling of nausea. When active, the patient has been experiencing dizzy spells and cannot stay on his feet for too long. John has found it hard to sleep due to an aching pain emanating from his inner right ear, yet he finds it difficult to communicate this pain.

In this instance, 2 of the 9 Questions contained in CoreQuestionnaire has its adaptive properties triggered - GenerallyUnwellCoreQuestion and EarCoreQuestion. The generally unwell Questionnaire includes 7 Questions, of which none are adaptive, meaning all are parsed by the system. On the other hand, EarQuestionnaire is made up of 9 Questions, with 4 of these being dependent on the user's ability to hear. Since John has indicated that he is deaf, these 4 Questions are not presented. Therefore, John is required to answer a total of 21 Questions $(19.09 \%)$.

\section{Conclusion \& Future Work}

We have proposed a model for an adaptive questionnaire for patients with learning disabilities that responds to both the accessibility needs of patients (physical and cognitive) as well as their clinical context. In that model, an individual's accessibility profile (i.e. the presence of impairments) is used to customize the interaction and interface to the user's needs. The model also adapts the structure and content of the questionnaire to the patient's specific clinical context to ensure that only relevant questions are asked or expanded on. This is the first research conducted to apply these technologies to the domain of medical data collection for patients with LDs. Opportunities for future work include: expanding our questionnaire to incorporate further conditions; implementing the functionality required to map the extracted accessibility profile to the elements included in the interface; adding further decision support functionalities as in [18] and conducting user evaluations to determine whether the system meets the care needs of patients with LDs and medical practitioners.

\section{References}

1. Bachman, J.W.: The Patient-Computer Interview: A Neglected Tool That Can Aid the Clinician. Mayo Clinic Proceedings. 78, 67-78 (2003). DOI: 10.4065/78.1.67

2. Afia, A. et al.: Discrimination and other barriers to accessing health care: perspectives of patients with mild and moderate intellectual disability and their carers. PloS one vol. 8,8 e70855 (2013). DOI: 10.1371/journal.pone. 0070855 
3. Bouamrane, M.-M., Rector, A., Hurrell, M.: Gathering Precise Patient Medical History with an Ontology-Driven Adaptive Questionnaire, 21st IEEE Symposium on Computer-Based Medical Systems, Jyvaskyla, 2008, pp. 539-541 (2008). DOI: 10.1109/CBMS.2008.24

4. Bouamrane, M.-M., Rector, A., Hurrell, M.: Ontology-Driven Adaptive Medical Information Collection System. In: Foundations of Intelligent Systems. pp. 574-584. Springer Berlin Heidelberg (2008). DOI: 10.1007/978-3-540-68123-6_62

5. Bouamrane MM, Rector A, Hurrell M. Using ontologies for an intelligent patient modelling, adaptation and management system. On the Move to Meaningful Internet Systems: OTM 2008, Volume 5332 of the series LNCS. p. 1458-70. DOI: 10.1007/978-3-540-88873-4_36

6. Gibson, R.C., Bouamrane, M.-M., Dunlop, M.: Mobile support for adults with mild learning disabilities during clinical consultations. MobileHCI '18. ACM Press, Spain (2018). DOI: 10.1145/3229434.3229469

7. Gibson, R.C., Bouamrane, M.-M., Dunlop, M.: Design Requirements for a Digital Aid to Support Adults with Mild Learning Disabilities During Clinical Consultations: Qualitative Study with Experts. JMIR Rehabilitation and Assistive Technologies. 6, e10449 (2019). DOI: $10.2196 / 10449$

8. Benmimoune, L., Hajjam, A., Ghodous, P., Andres, E., Hajjam, M.: Ontology-Based Contextual Information Gathering Tool for Collecting Patients Data Before, During and After a Digestive Surgery. In: Handbook of Large-Scale Distributed Computing in Smart Healthcare. pp. 623-635. Springer International Publishing, Cham (2017). DOI: 10.1007/978-3-319-58280-1_23

9. Yesilada, Y., Harper, S., Goble, C.A., Stevens, R.R.: Ontology Based Semantic Annotation for Enhancing Mobility Support for Visually Impaired Web Users. Proceedings of the KCAP 2003 Workshop on Knowledge Markup and Semantic Annotation (2003).

10. Obrenovic, Z., Starcevic, D., Devedzic, V.: Using ontologies in design of multimodal user interfaces. INTERACT 2003; Zurich, Switzerland. pp. 535-542. IOS Press (2003).

11. Castillejo, E., Almeida, A., López-de-Ipiña, D.: Ontology-Based Model for Supporting Dynamic and Adaptive User Interfaces. International Journal of Human-Computer Interaction. 30, 771-786 (2014). DOI: 10.1080/10447318.2014.927287

12. Karim, S., Tjoa, A.M.: Towards the Use of Ontologies for Improving User Interaction for People with Special Needs. In: Computers Helping People with Special Needs. pp. 77-84. Springer Berlin Heidelberg (2006). DOI: 10.1007/11788713_12

13. Karim, S., Tjoa, A.M.: Connecting User Interfaces and User Impairments for Semantically Optimized Information Flow in Hospital Information Systems. In: Proceedings of IMEDIA '07 and I-SEMANTICS '07 (2007).

14. Marino, B.D.R., Rodriguez-Fortiz, M.J., Torres, M.V.H., Haddad, H.M.: Accessibility and Activity-Centered Design for ICT Users: ACCESIBILITIC Ontology. IEEE Access. 6, (2018). DOI: 10.1109/ACCESS.2018.2875869

15. Happel, H.-J., Seedorf, S.: Applications of Ontologies in Software Engineering. In: Semantic Web Enabled Software Engineering (SWESE) (2006)

16. Knublauch, H., Fergerson, R.W., Noy, N.F., Musen, M.A.: The Protégé OWL Plugin: An Open Development Environment for Semantic Web Applications. In: The Semantic Web ISWC 2004. pp. 229-243. Springer Berlin Heidelberg, (2004). DOI: 10.1007/978-3-54030475-3_17

17. Horridge, M., Bechhofer, S., Noppens, O.: Igniting the OWL 1.1 touch paper: The OWL API. In: OWLED (2007)

18. Bouamrane, MM., Rector, A. Hurrell, M.: Using OWL ontologies for adaptive patient information modelling and preoperative clinical decision support. Knowl Inf Syst 29 pp.40518 (2011). DOI: $10.1007 / \mathrm{s} 10115-010-0351-7$ 